\title{
Accuracy Improvement of Neutron Nuclear Data on Minor Actinides
}

\author{
Hideo Harada ${ }^{1, a}$, Osamu Iwamoto ${ }^{1}$, Nobuyuki Iwamoto ${ }^{1}$, Atsushi Kimura ${ }^{1}$, Kazushi Terada ${ }^{1}$, Taro Nakao ${ }^{1}$ \\ Shoji Nakamura ${ }^{1}$, Kazuhito Mizuyama ${ }^{1}$, Masayuki Igashira ${ }^{2}$, Tatsuya Katabuchi ${ }^{2}$, Tadafumi Sano ${ }^{3}$, \\ Yoshiyuki Takahashi $^{3}$, Koichi Takamiya ${ }^{3}$, Cheol Ho Pyeon ${ }^{3}$, Satoshi Fukutani ${ }^{3}$, Toshiyuki Fujii ${ }^{3}$, Jun-ichi Hori ${ }^{3}$, Takahiro \\ Yagi $^{3}$ and Hiroshi Yashima ${ }^{3}$ \\ ${ }^{1}$ Japan Atomic Energy Agency, Nuclear Science and Engineering Center, 319-1195 Tokai-mura, Ibaraki, Japan \\ ${ }^{2}$ Tokyo Institute of Technology, Research Laboratory for Nuclear Reactors, 152-8550 O-okayama, Tokyo, Japan \\ ${ }^{3}$ Kyoto University, Research Reactor Institute, 590-0494 Kumatori-cho, Osaka, Japan
}

\begin{abstract}
Improvement of accuracy of neutron nuclear data for minor actinides (MAs) and long-lived fission products (LLFPs) is required for developing innovative nuclear system transmuting these nuclei. In order to meet the requirement, the project entitled as "Research and development for Accuracy Improvement of neutron nuclear data on Minor ACtinides (AIMAC)" has been started as one of the "Innovative Nuclear Research and Development Program" in Japan at October 2013. The AIMAC project team is composed of researchers in four different fields: differential nuclear data measurement, integral nuclear data measurement, nuclear chemistry, and nuclear data evaluation. By integrating all of the forefront knowledge and techniques in these fields, the team aims at improving the accuracy of the data. The background and research plan of the AIMAC project are presented.
\end{abstract}

\section{Introduction}

It was recognized by $\mathrm{OECD} / \mathrm{NEA} / \mathrm{WPEC}$ experts group SG-31 [1] that pertinent efforts of experiments and evaluations are still required and indispensable in order to meet the requirements [2] of accurate nuclear data for developing advanced nuclear systems including accerelator driven minor actinide burner [3]. For examples, there are large gaps on capture cross sections of minor actinides (MAs) between current uncertainty and required accuracy as summarized in Table 1, which are based on WPEC/SG-26 evaluation [2]. The required accuracy depends on the type of reactor system and the neutron energy region as shown in Table 1. ADMAB means an accelerator-driven minor actinides burner, GFR a gas-cooled fast reactor, and SFR a sodium-cooled fast reactor. Current uncertainties are 2-5 times larger than required accuracies for designing advanced nuclear systems.

It is understood that the experimental result is the best estimate of the value of the measurement, and that all components of uncertainty contribute to the total uncertainty. However, some of systematic effects are sometimes unrecognized and not discussed in published papers. It is worthwhile to notice that only the recognized systematic effects are corrected and took into account as a part of the total uncertainty. This explains why there is sometimes discrepancy between experimental results exceeding their uncertainties. It is

\footnotetext{
${ }^{\mathrm{a}}$ Corresponding author: harada.hideo@jaea.go.jp
}

important to solve the discrepancy by identifying unrecognized bias effect to achieve the required accuracy.

Table 1. Comparison between current uncertainty and required accuracy on MA's capture cross section.

\begin{tabular}{|c|c|c|c|c|}
\hline Nucleus & $\begin{array}{c}\text { Current } \\
\text { uncert. }\end{array}$ & $\begin{array}{c}\text { Required } \\
\text { accuracy }\end{array}$ & $\begin{array}{c}\text { Range } \\
\text { (keV) }\end{array}$ & $\begin{array}{c}\text { Reactor } \\
\text { Type }\end{array}$ \\
\hline${ }^{237} \mathrm{~Np}$ & $6 \%$ & $3 \%$ & $0.5-498$ & ADMAB \\
\hline${ }^{241} \mathrm{Am}$ & $8 \%$ & $\begin{array}{c}2 \% \\
3 \%\end{array}$ & $\begin{array}{c}0.5-1350 \\
2-183\end{array}$ & $\begin{array}{c}\text { ADMAB } \\
\text { GFR }\end{array}$ \\
\hline${ }^{242 \mathrm{~m}} \mathrm{Am}$ & $25 \%$ & $12 \%$ & $67-498$ & SFR \\
\hline${ }^{243} \mathrm{Am}$ & $10 \%$ & $2 \%$ & $0.5-1350$ & ADMAB \\
\hline${ }^{244} \mathrm{Cm}$ & $20 \%$ & $6 \%$ & $9-498$ & ADMAB \\
\hline
\end{tabular}

Recently, there are striking technical advancements in nuclear data measurement methods. Especially, highintensity-pulsed neutrons generated by spallation reaction become available to obtain high precision neutron TOF data. By utilizing the advantage, research programs are going on to measure capture cross sections of highly radioactive nuclei at CERN [4], LANL [5], and J-PARC. 
As an prominent example, capture cross section of highly radioactive nucleus ${ }^{244} \mathrm{Cm}\left(\mathrm{T}_{1 / 2}=18 \mathrm{yr}\right)$ was measuerd [6] at neutron TOF beam line ANNRI (Accurate NeutronNucleus Reaction Measurement Instrument) installed at J-PARC/MLF [7]; there is only one experiment using nuclear explosion method [8] before the measuement at ANNRI. The other important advantage is to be able to use thin samples. This makes correction factor extreamly small on neutron self-absorption and multiple-scattering effect in a sample while keeping enough statistics as demonstrated in the case of ${ }^{241} \mathrm{Am}$ [9] at ANNRI/J-PARC.

In order to deduce an accurate nuclear data from high precision TOF data, accurate normalization is one of the most important issues. The double check experiments are valuable for identifying unrecognized bias effect, and integral experiments to verify the results are indispensable.

In order to meet the requirement, the project entitled as "Research and development for Accuracy Improvement of neutron nuclear data on Minor ACtinides (AIMAC)" has been started as one of the "Innovative Nuclear Research and Development Program" in Japan at October 2013. The AIMAC project team is composed of researchers in four different fields: differential nuclear data measurement, integral nuclear data measurement, nuclear chemistry, and nuclear data evaluation. By integrating all of the forefront knowledge and techniques in these fields, the team aims at improving the accuracy of the data. The principal idea and research plan of the AIMAC project are presented in chapter 2 .

\section{Research Plan and Progress}

The following five research items have been conducted by the AIMAC project team: i) the thermal neutron capture cross sections, which are used for normalization of TOF data, will be measured for ${ }^{237} \mathrm{~Np},{ }^{241,243} \mathrm{Am}$ using activation method. The obtained results will be cross checked by integral measurements using variable and characterized neutron flux fields that will be developed in this project. ii) the amount of MA in samples used for TOF measurement will be determined by utilizing two independent methods. Their isotopic purity will be also examined by mass spectroscopic method. iii) low-energy resonances will be used for normalization. In order to deduce resonance parameters, neutron transmission and capture experiments will be performed using different thickness samples. iv) energy region of neutron capture data at ANNRI will be extended to a few hundred keV region. v) high quality evaluation will be performed based on iterative communication with experimenters.

Independent double check experiments and close communication between different research fields are special features of the AIMAC project toward accuracy improvement of neutron nuclear data. The research plan and some progress are summarized below.

\subsection{Accurate measurements of thermal neutron capture cross-sections}

In order to determine neutron capture cross sections using activation methods, $\alpha$ or $\gamma$ spectroscopic method has been utilized in case of $\alpha$-ray emitting nucleus. In this project, both $\alpha$ and $\gamma$ spectroscopic methods will be utilized, and the deduced independent results will be compared.

To deduce accurate cross sections by $\gamma$ spectroscopic method, precise and accurate emission probability of decay $\gamma$ ray emitted from neutron captured nucleus and/or its decay product is required. In this project, the relevant emission probability of decay $\gamma$ ray is in progress.

Kyoto university reactor (KUR) is used for activation experiments. KUR is usually operated at 1-MW power with $20 \%$ low-enriched uranium fuel. During a period of irradiation for Boron Neutron Capture Therapy (BNCT), the maximum thermal power is $5 \mathrm{MW}$, and its thermalneutron flux is $8.2 \times 10^{13} \mathrm{n} / \mathrm{cm}^{2} / \mathrm{s}$ [10]. The experimental facilities available at KUR are as follows: the heavy water neutron irradiation facility, graphite thermal column, neutron guide tube, hydraulic conveyer, pneumatic tubes, slant exposure tube, and so on.

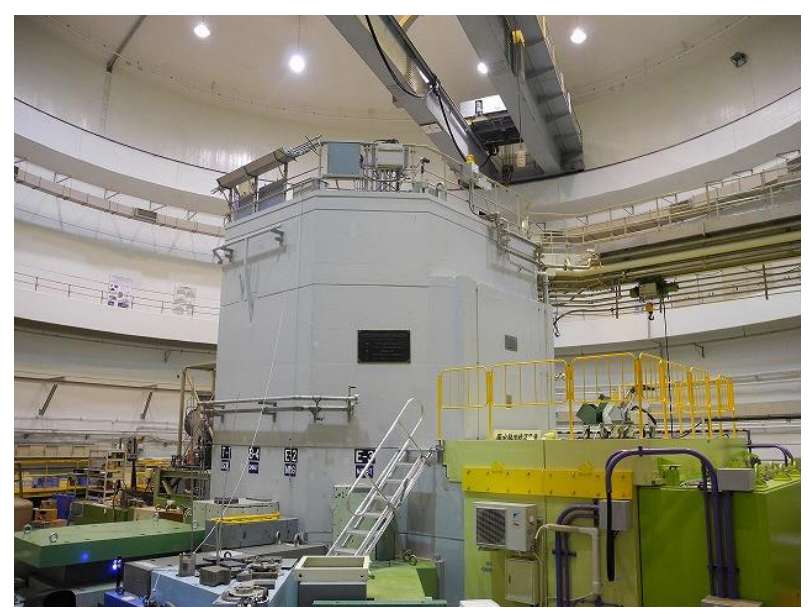

Figure 1. A photo of KUR.

\subsubsection{Test of evaluated data using variable neutron flux}

Neutron capture cross sections will be cross checked by integral measurements using variable and well characterized neutron flux fields. For developing these neutron fields, Kyoto University Critical Assembly (KUCA) and electron linear accelerator (KUR linac) based neutron source are utilized.

KUCA has been used for reactor physics experiments and its neutron flux is well characterized and its flux distribution is variable [11].

KUR linac has been used for nuclear data measurements using TOF method. Neutrons produced by photonuclear reactions were moderated by water. In this project, borated water moderator is used to supply variable neutron flux distribution. The neutron flux distribution is measuerd by TOF method.

\subsubsection{Combination of $\alpha$ and $y$ spectroscopy}




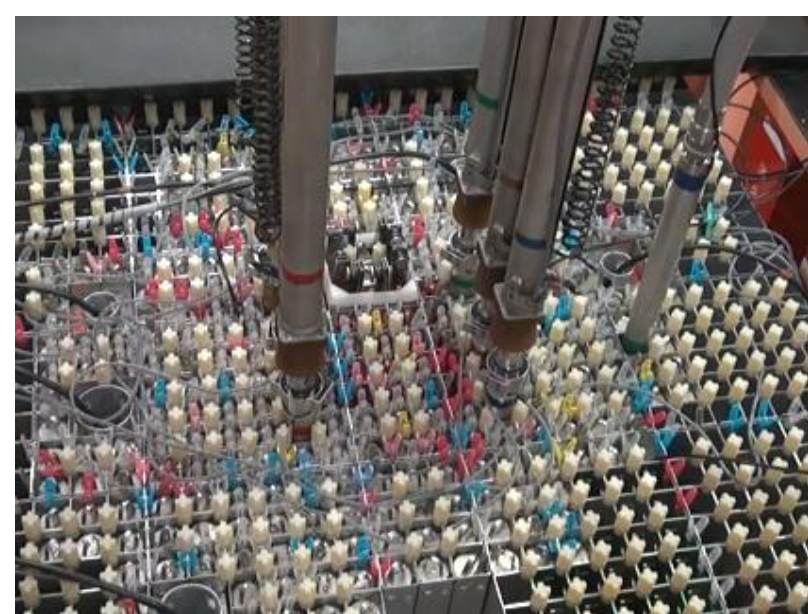

Figure 2. A photo of KUCA.

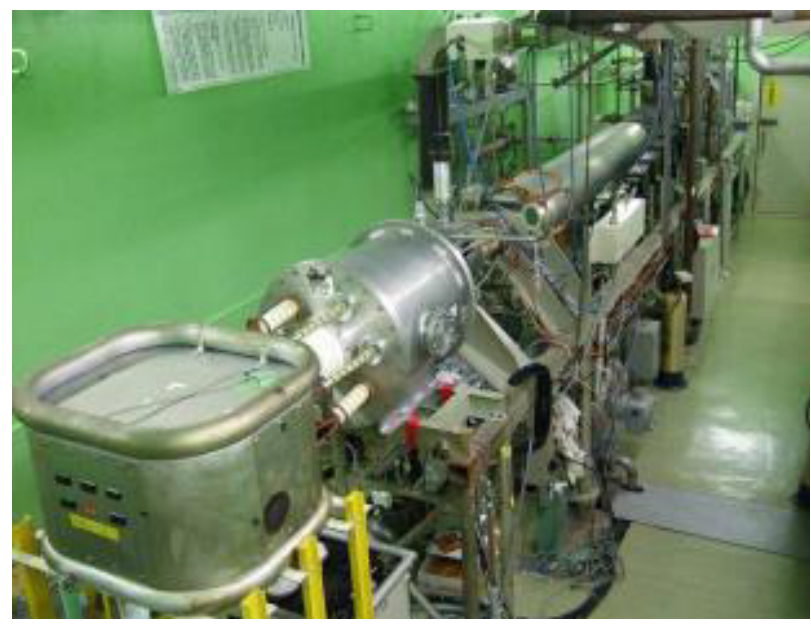

Figure 3. A photo of KUR linac.

\subsection{High-precision quantification of sample amount used for TOF measurement}

The amount of MA in samples shielded in capsule materials used for TOF measurements need to be determined precisely for absolute normalization of energy dependent cross sections. However, accurate quantification of the amount is sometimes difficult. In this project, these quantities are measured by utilizing two independent methods; $\gamma$-ray spectroscopic method and calorimetric method. Isotopic purity of the TOF sample is also examined by mass spectroscopic method.

\subsubsection{Determination of decay $\mathrm{y}$-ray intensity}

To determine the sample amount non-destructively, decay $\gamma$ rays from the MA sample can be used with $\gamma$ ray spectroscopy in principle. However, accurate $\gamma$ ray emmision probability is necessary to be precisely determined. In this project, the relevant $\gamma$ ray emmision probabilities for MA samples are measuerd using a combination of $\alpha$-ray and $\gamma$-ray spectroscopic methods [12].

${ }^{237} \mathrm{~Np}$ and ${ }^{241,243} \mathrm{Am}$ standard solutions were supplied by Japan Radioisotope Association to prepare samples. A Si detector is utilized to determine the activity of the sample by counting alpha particles. The $\gamma$ rays emitted from the samples are measured with a high purity $\mathrm{Ge}$ spectrometer. The $\gamma$-ray emission probability is derived from the activity and the $\gamma$ ray yield. The measurements with high precision (within $2 \%$ ) are in progress.

\subsubsection{Utilization of Micro Calorimetry}

Direct heat measurement can be also used to determine the sample amount non-destructively, since the $\mathrm{Q}$ values of $\alpha$-ray emmision and half-lives are much precicely determined relative to $\gamma$-ray emission probability. Recent micro calorimeter has a potential to measure heat with precision of sub $\mu$-Watt. As an example, the decay heat by a $1 \mathrm{GBq}^{241} \mathrm{Am}$ sample is about $900 \mu \mathrm{W}$. In this case, it is expected to be able to determine the sample amount with precision less than about $0.1 \%$ in principle by direct heat measurement. We are planning to introduce microcalorimeter, which has potential to determine heat deposit with $100 \mathrm{nW}$ accuracy and has a volume of $20 \mathrm{ml}$ sample cell.

In order to evaluate the amount of heat loss by decay gamma-rays escaping to outside of the cell, Monte-Carlo simulation study has been performed. In case of ${ }^{241} \mathrm{Am}$, decay gamma-rays can be well shielded by setting $1 \mathrm{~cm}$ thick $\mathrm{Pb}$ pellets under and over the sample. In case of ${ }^{243} \mathrm{Am}$, escaping heat due to outgoing gamma-ray is about $0.3 \%$ relative to the total heat deposit. It is expected the fine correction for escaping heat can be done by the Monte-Carlo simulations developed in this work.

As the other bias effect, heat deposit by radioactive contaminants need to be considered. The combination of gamma-ray measurement is expected to solve the issue.

\subsubsection{Preparation of MA samples}

Different thickness MA samples are prepared for TOF measurements. By TOF measurements using different samples, identification of unrecognized origin of systematic error is expected, and also the samples are used for resonance parameter determination. It is important to know isotopic purity in the samples since the contribution of contaminated isotopes needs to be corrected.

For analyses of materials used for the sample preparation, a portion of the same materials used for the sample has been analyzed by utilizing destructive method; mass, $\alpha$-ray and $\gamma$-ray spectroscopic methods. Thermal ionization mass spectrometer (TIMS) at KUR is used, which has potential to determine isotopic ratios with precision of $0.1 \%$ or less.

\subsection{Resonance parameter determination by combining total and capture cross sections}

\subsubsection{Measurements at ANNRI}

Neutron capture cross section measuements at the TOF beam line ANNRI at J-PARC/MLF (figure 4) have been 
performed for ${ }^{237} \mathrm{~Np}$ [13], ${ }^{241} \mathrm{Am}$ [9] and ${ }^{244,246} \mathrm{Cm}$ [6] on MAs using high resolution $\gamma$-ray spectrometers. In order to deduce resonance parameters precicely, neutron total cross section measurements are planned in this project, and Li glass neutron detector is under development. At least, two kind of thickness MA samples are used for neutron transmission measurements.

Current proton beam power generating spallation neutrons is $300 \mathrm{~kW}$, and supplying a world highest flux neutron beam for TOF measurement. During the AIMAC project period, the beam power is planned to be increased about twice.

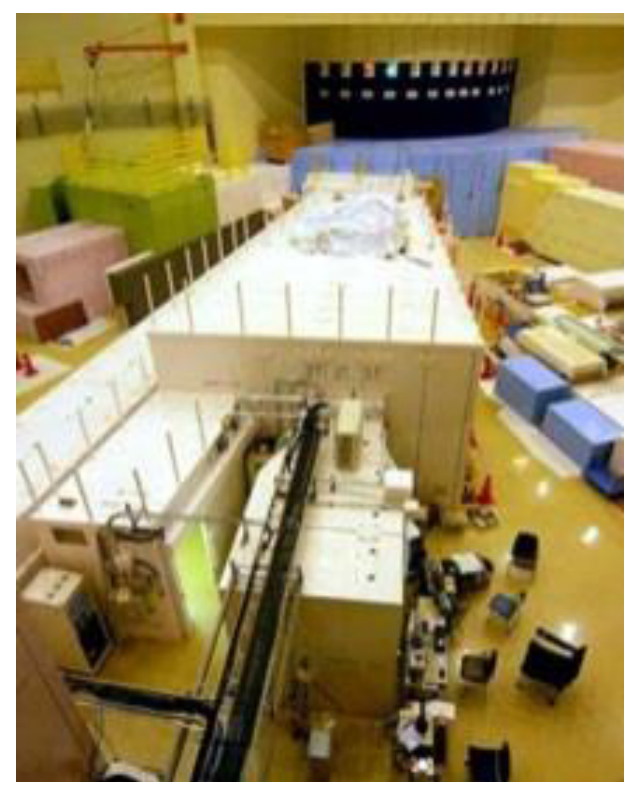

Figure 4. A photo of J-PARC / ANNRI.

\subsubsection{Measurements at KUR linac}

Neutron capture cross section measuements at the TOF beam line of KUR-linac (figure 3) have been performed for various samples including ${ }^{237} \mathrm{~Np}[14,15]$ and ${ }^{243} \mathrm{Am}$ [16]. The short pulse-width electron beam is available from 2 nsec. This enebles high resolution neutron TOF spectroscopy, and supplies complementary data to JPARC/ANNRI data. High efficiency BGO spectrometer will be developed and installed at $10 \mathrm{~m}$ TOF station. Neutron total cross section measurements are also planned in this project by using Li glass neutron detector installed at $22 \mathrm{~m}$ TOF station. In order to optimize the neutron beam quality, we are designing a neutron moderator and collimators.

\subsection{Extension of capture cross section measurements to high energy region}

$\mathrm{NaI}(\mathrm{Tl})$ detectors installed in ANNRI are used for neutron capture cross section measurements in the high energy region. Faster time response of $\mathrm{NaI}(\mathrm{Tl})$ detectors than Ge detectors allows measurements in the high energy region. The pulse-height weighting technique using an $\mathrm{NaI}(\mathrm{Tl})$ detector has been well established by the Tokyo Tech group [17]. The NaI(Tl) detectors consist of two differentially-sized detectors, placed in the downstream area of ANNRI (Fig. 5). The details of the detector system can be found in ref. [18]. The sample position is set at a flight path length of $27.9 \mathrm{~m}$.

The current high energy limit of measurements with the ANNRI-NaI(Tl) detectors is about $100 \mathrm{keV}$. Modification of the detector system to extend the high energy limit to about $300 \mathrm{keV}$ is underway. In order to perform measurements in the high energy region, we need to overcome two problems: $\gamma$-flash and fast neutron background. Gamma-ray burst called $\gamma$-flash is emitted from the spallation neutron source at the beginning of each TOF cycle. The $\gamma$-flash induces a large detector output, saturating electronics and data acquisition system in certain duration of time after the $\gamma$-flash. To minimize the saturation, we replaced an old-style pulse height analysis system with a high speed time digitizer. Analog electronics modules such as shaping amplifiers were removed. Instead of measuring the pulse height with the conventional peak-hold analog-to-digital convertor, a new method to calculate the pulse height from the pulse width measured with the time digitizer was developed [18]. To lower the fast neutron background, we are designing neutron shields for the detectors.

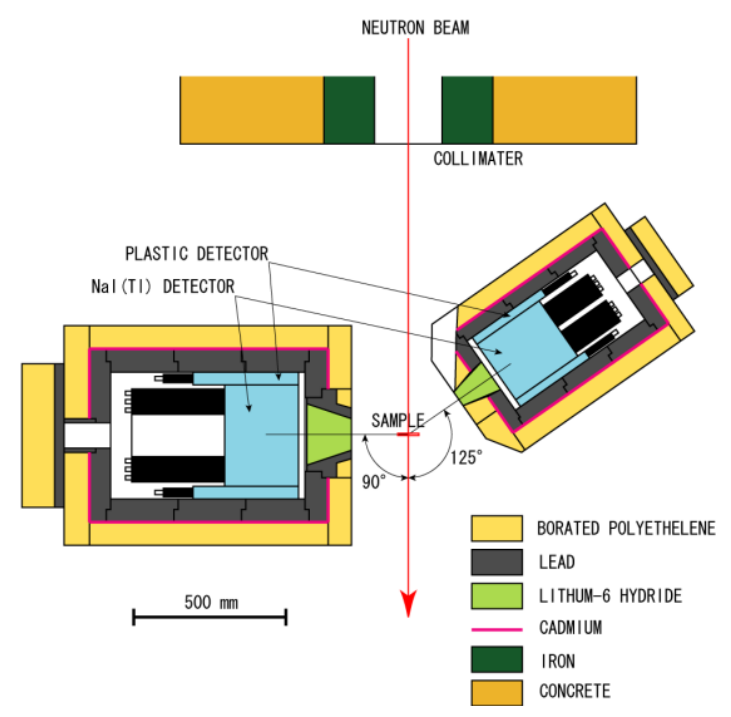

Figure 5. $\mathrm{NaI}(\mathrm{Tl})$ detectors of ANNRI.

\subsection{High quality evaluation based on iterative communication with experimenters}

To investigate the present accuracy, available experimental data for some of important MAs and FPs were reviewed. Based on them, it is concluded that the uncertainties of the MA capture cross sections in the upto-date Japanese evaluated nuclear data library JENDL4.0 [19] are in consistency with the difference between evaluation and experimental data, and seem to be reasonable.

On the other hand, there are no covariance data of well-known long-lived fission products ${ }^{99} \mathrm{Tc}$ and ${ }^{129} \mathrm{I}$ in JENDL-4.0. At present, the two nuclides are targeted for nuclear transmutation. In order to estimate the 
uncertainty of their transmutation efficiencies, covariance data of their capture cross sections are needed in the $\mathrm{keV}$ energy region. Therefore, covariance evaluations were performed with nuclear reaction calculation code CCONE [20] and covariance evaluation code KALMAN [21]. In order to derive the covariance, we set initial uncertainties for $\sim 50$ parameters (e.g., optical model potential, gamma-ray strength function, and level density) in nuclear reaction models, so as to cover the area wider than the spread of experimental data. In the case of ${ }^{99} \mathrm{Tc}$, the cross section uncertainty obtained from the assumed parameter uncertainties is shown by the shaded light gray area in Fig. 6. In this evaluation representative uncertainties were considered as shown by error bars without symbol in Fig. 6, instead of making use of experimental data directly. The calculated uncertainty is $12-18 \%$ whose range is illustrated by shaded dark gray area in Fig. 6, and marginally includes the data of Macklin and Winters [22] and Kobayashi et al [23].

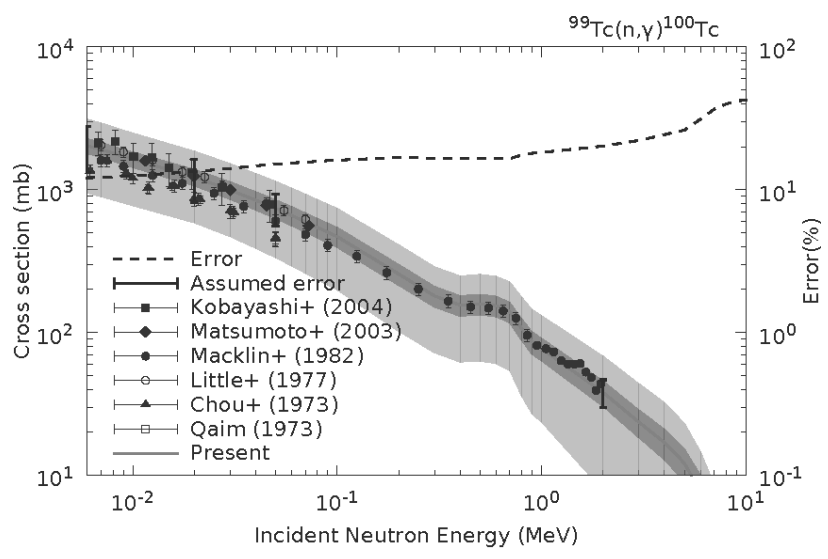

Figure 6. Evaluated uncertainty of capture cross section on ${ }^{99} \mathrm{Tc}$ compared with experimental data. Shaded light and dark gray areas represent a prior error based on an assumed uncertainty of parameters and posterior one, respectively.

\section{Conclusions}

Japanese nuclear data project entitled as "Research and development for Accuracy Improvement of neutron nuclear data on Minor ACtinides (AIMAC)" has been outlined. It was pointed out that high precision TOF data is becoming available by using a recent state-of-art pulsed neutron facility such as ANNRI at J-PARC although there is still issue on data normalization. The importance of identifying unrecognized systematic bias effect is also pointed for accuracy improvement. Aiming at satisfying accuracy requirements for designing nuclear transmutation systems, experts in four different fields (nuclear data measurement, nuclear data evaluation, reactor physics, and nuclear chemistry) made a research plan of AIMAC to overcome the normalization issue and identify unrecognized bias effect.

Recently, OECD/NEA/WPEC approved a new subgroup SG-41 titled as "improving nuclear data accuracy of ${ }^{241} \mathrm{Am}$ and ${ }^{237} \mathrm{~Np}$ capture cross sections (INDA)". Expert's knowledge on evaluations, energy dependent methods, spectrum averaged methods, nuclear structure data related to capture cross sections are integrated internationally for accuracy improvement of nuclear data. AIMAC project is expected to contribute to this internatinally recognized issue on accuracy improvement of nuclear data.

\section{Acknowledgement}

Present study includes the result of "Research and Development for accuracy improvement of neutron nuclear data on minor actinides" entrusted to the Japan Atomic Energy Agency by the Ministry of Education, Culture, Sports, Science and Technology of Japan (MEXT).

\section{References}

1. H. Harada et al., WPEC-31, OECD NEA (2014)

2. M. Salvatores et al., WPEC-26, OECD NEA (2008)

3. K. Tsujimoto, Proc. 2012 Symposium on Nuclear Data, JAEA-Conf 2013-002, p.71 (2013)

4. U. Abbondanno et al. (n_TOF Collaboration), Phys. Rev. Lett. 93, 161103 (2004)

5. M. Jandel et al., Phys. Rev. C 78, 034609 (2008)

6. A. Kimura et al., J. Nucl. Sci. Technol. 49, 708 (2012)

7. Y. Kiyanagi et al., J. Korean Phys. Soc. 59, 1781 (2011).

8. M.S. Moore, G.A. Keyworth, PRC 3, 1656 (1971)

9. H. Harada et al., Nucl. Data Sheets 119, 61 (2014)

10. T. Sano et al., Proc, PHYSOR2014 (2014) (to be published)

11. H. Unesaki, Doctor thesis (2001)

12. H. Harada et al., J. Nucl. Sci. Technol. 43, 1289 (2006)

13. K. Hirose et al., J. Nucl. Sci. Technol. 50, 188 (2013)

14. O. Shcherbakov et al., J. Nucl. Sci. Technol. 42, 135 (2005)

15. M. Mizumoto et al., Proc. Int. Conf. on Nuclear Data for Science and Technol., April 22-27, 2007, Nice, France, pp. 591 (2008)

16. J. Hori et al., JAEA-Conf 2009-004, 123 (2009)

17. S. Mizuno, et al., J. Nucl. Sci. Technol. 36, 493 (1999)

18. T. Katabuchi et al., Nucl. Instrum. Meth. A 764, 369, (2014)

19. K. Shibata et al., J. Nucl. Sci. Technol. 48, 1 (2011)

20. O. Iwamoto, J. Nucl. Sci. Technol. 44, 687 (2007)

21. T. Kawano and K. Shibata, JAERI-Data/Code 97037, Japan Atomic Energy Research Institute (1997)

22. R.L. Macklin and R.R. Winters, Nucl. Sci. Eng. 81, 520 (1982)

23. K. Kobayashi et al., Nucl. Sci. Eng. 146, 209 (2004) 
